\title{
Effective handover communication: Do we need more evidence?
}

\author{
Roy Ilan MD MSc FRCPC
}

\begin{abstract}
$\mathrm{T}^{\mathrm{s}}$ he transition of a patient from the intensive care unit (ICU) to a hospital ward is one of the more complex handover scenarios in health care, and one that carries a host of potential hazards for patients. The decreased level of supervision and monitoring on the ward may result in delays in identifying and managing urgent problems. The available resources, as well as non-ICU providers' clinical skill and expertise, may fail to meet the patient's needs. Clinical teams would typically have limited information about, and experience with, the patient; this lack of reference point may result in failure to recognize and respond to meaningful clinical changes. Furthermore, management plans developed while the patient was in the ICU risk not being implemented as intended. As a result, ICU survivors and their families are commonly left with a sense of abandonment, mistrust of the hospital system, fear and anxiety.

An effective handover process between providers in the ICU and on the ward can address various sources of harm, and foster a patientcentred continuity of care. However, because methodological limitations are very common in the published literature, how to make handover effective is yet to be explored (1). Mounting evidence suggests that improving handover communication requires a cultural change and a multifaceted strategy, including education, appropriate supervision of trainees (when applicable), integration of electronic and other tools into providers' workflow, and a restructuring of routine processes (2). However, establishing such changes in a given system outside of a funded research context, and sustaining them over time, is very challenging.

In the current issue of the Journal, Li et al (3) (pages 109-118) describe the perceptions of Canadian ICU administrators, as well as very small samples of ICU providers from Canada, the United States and the United Kingdom, regarding routine discharge practices in their hospitals. The responses indicate substantial variability among ICUs in discharge processes, an overall perceived mediocre quality of discharge practices and much-needed insights regarding improvement.

Generally, self-reporting in surveys conducted for studies of this nature frequently exceeds the actually practiced behaviour. Therefore, the extent of the problem is likely greater than portrayed in this study: the effectiveness of communication between relevant stakeholders around ICU discharge is likely suboptimal for many critically ill patients. Handover communication failures occasionally result in preventable patient safety incidents.
\end{abstract}

Additional research may shed light on different aspects of this problem. It is important to explore how specific interventions can address the needs of the different stakeholders involved, and can ensure a seamless transition of care and optimal management of patients' acute and chronic conditions post-ICU discharge.

However, I would strongly recommend against waiting for 'golden' scientific evidence before taking action. It is difficult to establish the cause-effect relationship between communication failures and downstream safety incidents. Similarly, substantial methodological limitations are inherent to the study of handover.

Nevertheless, there is consensus about the importance of an effective handover process. The real challenge has more to do with system and behavioural adaptation than with the available scientific evidence. Change is needed on the ground - not in the academic ivory towers of study and research.

In this case, quality improvement strategies are the solution. ICU leaders with intimate knowledge of their specific environments should design, incrementally implement, and evaluate the effectiveness of interventions to ensure that handover occurs consistently on ICU discharge; that the preferred in-person communication is supported by concise written materials; that supporting electronic and other tools, such as checklists and reminders, are user-centred; and that the various needs of each stakeholder, specifically patients and their families, are addressed in the process. Locally available resources should be used and barriers should be eliminated. The importance of continuity of care after ICU discharge cannot be overemphasized. Li et al show us that there is a lot of work ahead.

\section{REFERENCES}

1. Horwitz LI. Does improving handoffs reduce medical error rates? JAMA 2013;310:2255-6.

2. Starmer AJ, Keohane CA, Lipsitz SR, et al. Changes in medical errors after implementation of a handoff program. N Engl J Med 2014;371:1803-12.

3. Li P, Boyd JM, Ghali WA, Stelfox HT. Stakeholder views regarding patient discharge from intensive care: Suboptimal quality and opportunities for improvement. Can Respir J 2015;22:109-18.

Department of Medicine, Queen's University, Kingston, Ontario

Correspondence: Dr Roy Ilan, Department of Medicine, Queen's University, 94 Stuart Street, Etherington Hall, Room 1005, Kingston,

Ontario K7L 3N6. Telephone 613 484-8877, e-mail ilanr@queensu.ca 


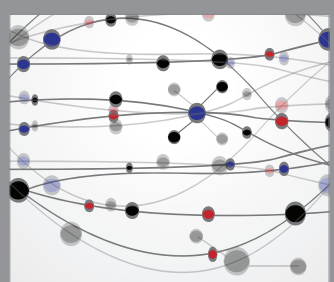

The Scientific World Journal
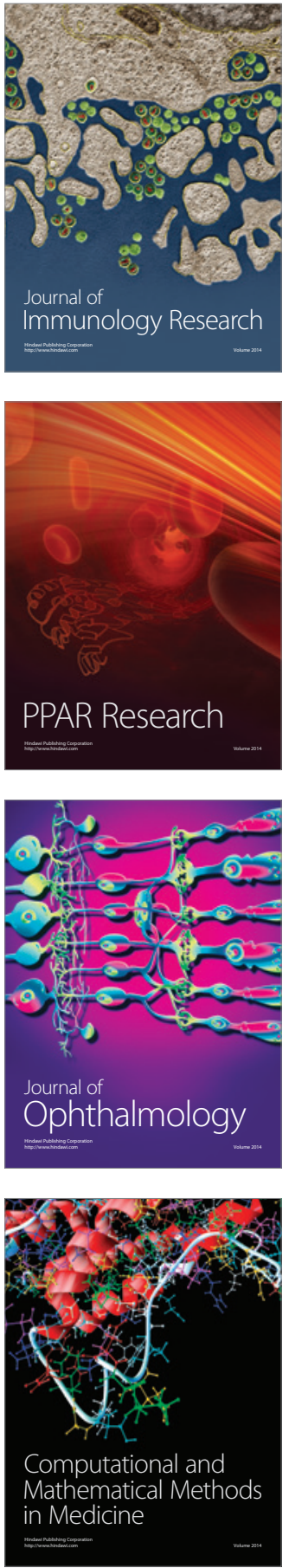

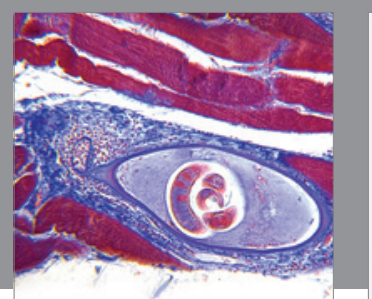

Gastroenterology Research and Practice

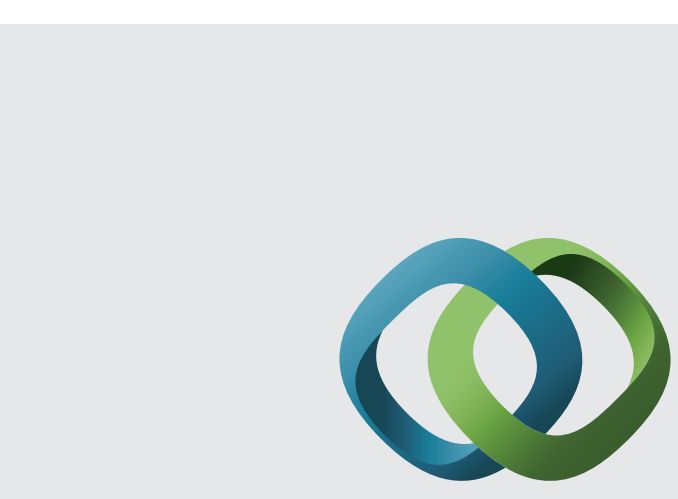

\section{Hindawi}

Submit your manuscripts at

http://www.hindawi.com
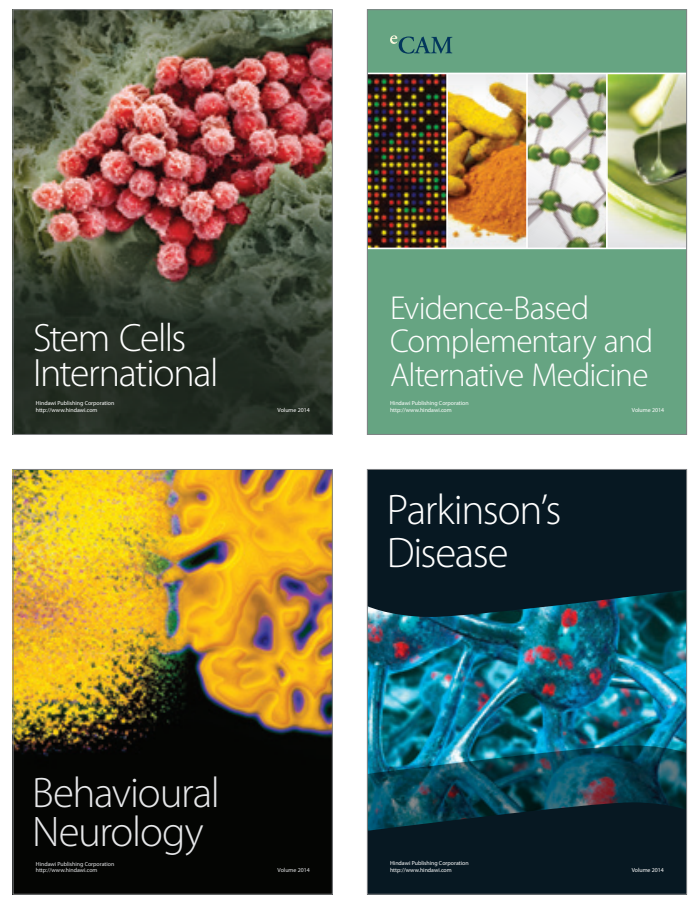
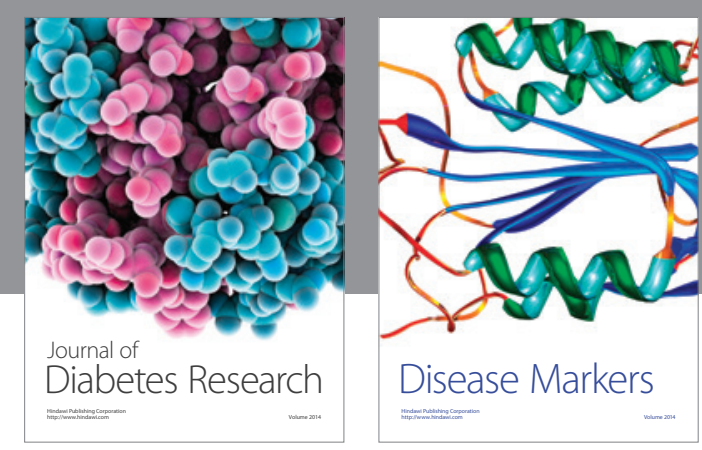

Disease Markers
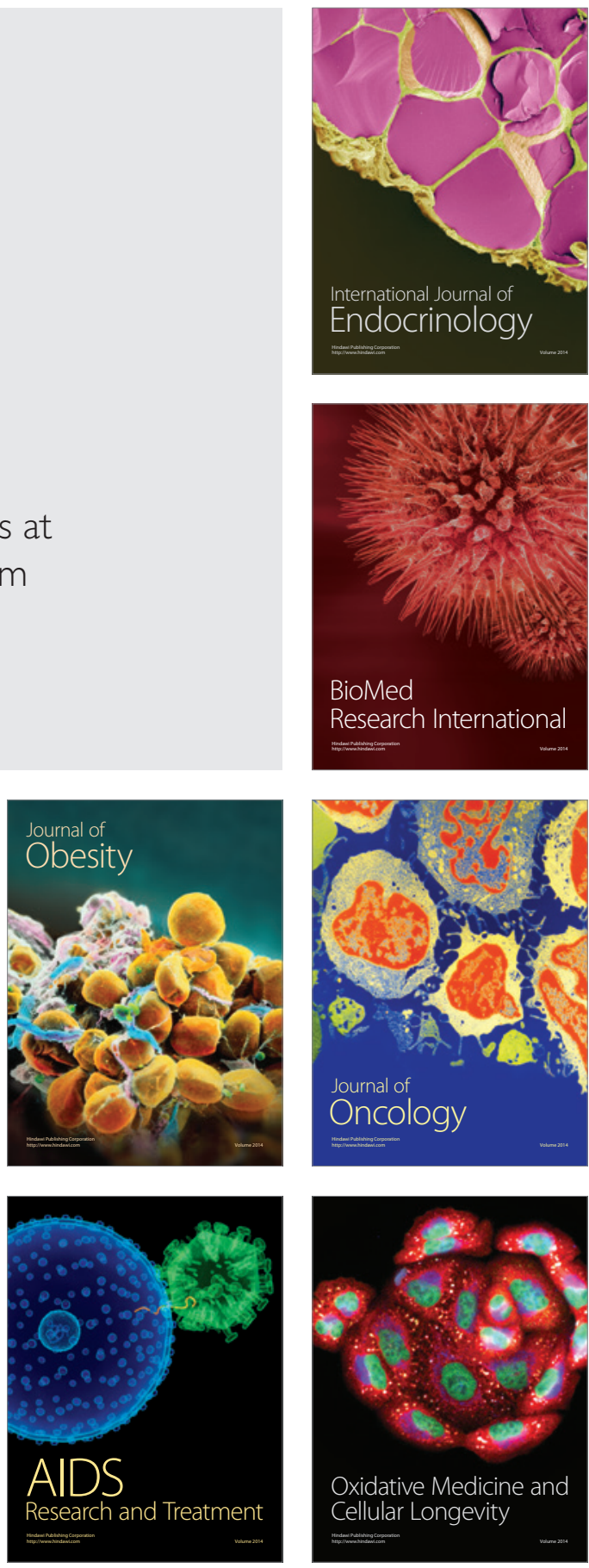